\title{
Exostosis costal como causa infrecuente de hemotórax. A propósito de un caso
}

\section{Costal exostosis as an infrequent cause of hemothorax. Case report}

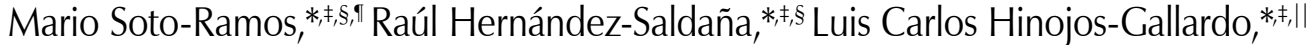 \\ Marcela Treviño-Zúñiga, ${ }^{* \neq}$ Noé Guerrero-Lucio, ${ }^{* \neq}$ Luis Roberto Rascón-Díaz, Ernesto Ramos-Martínez, ${ }^{* *}$ \\ Ruth Yazmín García-Sáenz, ${ }^{\mathbb{I}, \neq}$ Carlos Alberto Ríos-Contreras, ${ }^{\neq \neq}$Cristal Ariadne Salazar-Ortíz*

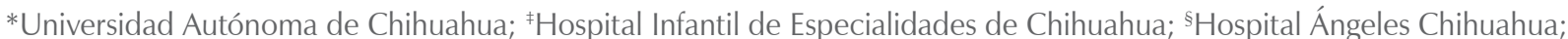 \\ "Instituto Municipal de Pensiones de Chihuahua; "Hospital Star Médica; **Laboratorio de Patología, Chihuahua; \\ ${ }^{\ddagger}$ Sanatorio Palmore de la Ciudad de Chihuahua.
}

RESUMEN. Los osteocondromas o exostosis osteocartilaginosas son las neoplasias benignas más frecuentes del tórax, pese a ello son excepcionales. Pueden encontrarse lesiones aisladas o múltiples, formando parte de un trastorno genético llamado exostosis cartilaginosa múltiple hereditaria. El hemotórax espontáneo es una presentación inusual de las exostosis costales. Se describe el caso de un adolescente de 13 años de edad con hemotórax espontáneo secundario a exostosis costal demostrada por técnicas de imagen. Se realizó tratamiento quirúrgico mediante toracoscopia y toracotomía con resección de la lesión.

Palabras clave: Osteocondromas, exostosis, costal, hemotórax.

\section{INTRODUCCIÓN}

El hemotórax es la presencia de sangre en la cavidad pleural definida por un hematócrito en líquido pleural mayor de 50\% del hematócrito sanguíneo. La mayoría de los hemotórax son secundarios a traumatismos naturales o iatrogénicos, con menor frecuencia se deben a una serie de patologías no traumáticas como la rotura de aneurisma aórtico, metástasis pleurales, anticoagulación por enfermedad tromboembólica y en un reducido número de casos el hemotórax catamenial. Se

Correspondencia:

Dr. Mario Soto-Ramos

Chihuahua, Chihuahua, México,

Correo electrónico: msoto67@gmail.com

Trabajo recibido: 13-VI-2020; aceptado: 12-VIII-2020.

Citar como: Soto-Ramos M, Hernández-Saldaña R, Hinojos-Gallardo LC, Treviño-Zúñiga M, Guerrero-Lucio N, Rascón-Díaz LR et al. Exostosis costal como causa infrecuente de hemotórax. A propósito de un caso. Neumol Cir Torax. 2020; 79 (4): 261-264. https://dx.doi.org/10.35366/97969
ABSTRACT. Osteochondromas or osteocartilaginous exostoses are the most frequent benign neoplasms of the chest, despite which they are exceptional. Isolated or multiple lesions can be found, forming part of a genetic disorder called-hereditary multiple cartilage exostosis. Spontaneous hemothorax is an unusual presentation of rib exostoses. We describe the case of a 13-year-old male adolescent with spontaneous hemothorax, secondary to rib exostosis, demonstrated by imaging techniques. Surgical treatment was performed by thoracoscopy and thoracotomy with resection of the lesion.

Keywords: Osteochondromas, exostosis, rib, hemotrax.

han descrito otras causas excepcionales de hemotórax no traumático, entre las que se encuentra la exostosis costal, aislada o en relación a la exostosis múltiple hereditaria. Presentamos un caso de hemotórax en un paciente joven, secundario a exostosis costal como parte de exostosis múltiple familiar.

\section{PRESENTACIÓN DEL CASO}

Se presenta el caso de un adolescente de 13 años de edad, estudiante, con antecedente patológico relevante de enfermedad de Ollier. Antecedente heredofamiliar de importancia, madre operada de osteocondroma en tibia durante su adolescencia. Acude a urgencias por presentar dolor torácico y disnea de inicio súbito de dos horas de evolución, sin antecedente de lesión traumática o antecedentes subyacentes.

A la exploración física somatométrica con peso de $45 \mathrm{~kg}$, talla $154 \mathrm{~cm}$, IMC $18.7 \mathrm{~kg} / \mathrm{m}^{2}$; T, $37{ }^{\circ} \mathrm{C}$; FC, 115x; FR, 26x; $\mathrm{SatO}_{2}, 92 \%$; consciente, orientado, cooperador, normocéfalo, faringe normal, cuello cilíndrico sin adenomegalias; tórax normolíneo con amplexión y amplexación disminuida en hemitórax derecho, con mate a la percusión, ruidos 
respiratorios ausentes en hemitórax derecho, hemitórax izquierdo con ruidos respiratorios normales; abdomen normal, extremidades íntegras, retorno capilar dos segundos, y neurológico íntegro.

En la radiografía de tórax simple (Figura 1) se aprecia una opacidad homogénea periférica en hemitórax derecho, con borramiento del ángulo costofrénico, concluyendo derrame pleural. El ultrasonido torácico demuestra derrame pleural no loculado.

Se realiza tomografía axial computarizada simple en la que se observó una lesión exostósica en la cuarta costilla del hemitórax derecho y derrame pleural (Figura 2A), y otra lesión exostósica en la sexta costilla del hemitórax izquierdo (Figura 2B). La densidad del derrame pleural derecho se describe en 60 unidades Hounsfield, sugiriendo ser un hemotórax. Se realiza toracocentesis del hemitórax derecho con catéter «único» Redax ${ }^{\circledR}$ con llave de tres vías, obteniendo 1,500 $\mathrm{mL}$ de sangre no coagulada. El citoquímico se reporta con $\mathrm{pH}$ de 8 , eritrocito y hemoglobina de 4+, sin células leucocitarias ni polimorfonucleares.

Se realiza toracoscopia del hemitórax derecho con pleurotomía cerrada a nivel del quinto espacio intercostal y línea axilar posterior, se visualiza en superficie pleural lesión exostósica en la cuarta costilla, con un coágulo de aproximadamente $50 \mathrm{~cm}^{3}$ y sangrado mínimo en capa de la misma lesión, sugiriendo ser la causa del hemitórax (Figura 3A). Se observan también múltiples exostosis costales del mismo hemitórax, con integridad de la pleura y sin sangrado evidente en otras zonas (Figura 3B).

Posteriormente se realizó toracotomía subaxilar derecha hasta llegar a la lesión exostósica, misma que fue resecada por completo junto con parte de la cuarta costilla a través de resección subperióstica.

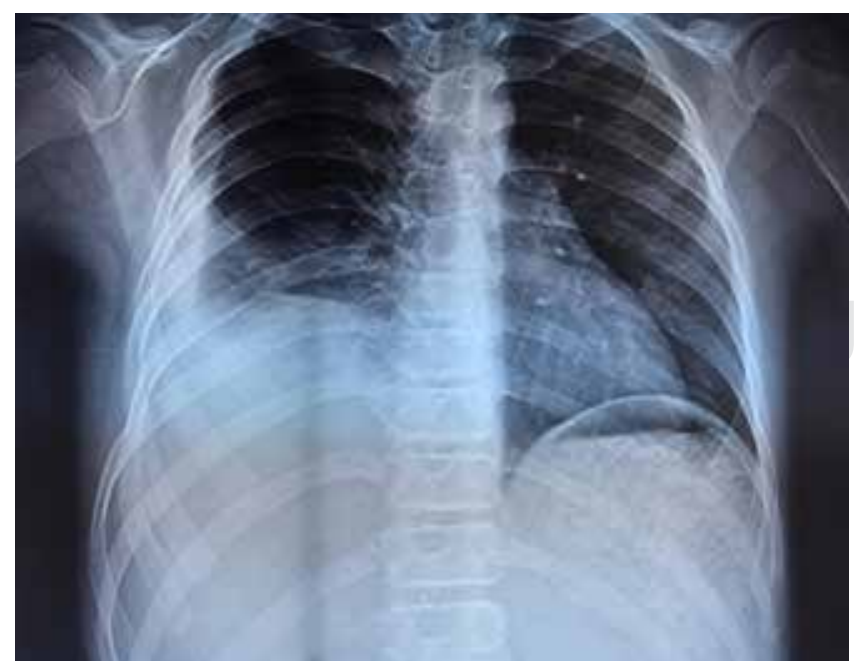

Figura 1: Radiografía posteroanterior en bipedestación con evidencia de derrame pleural derecho.
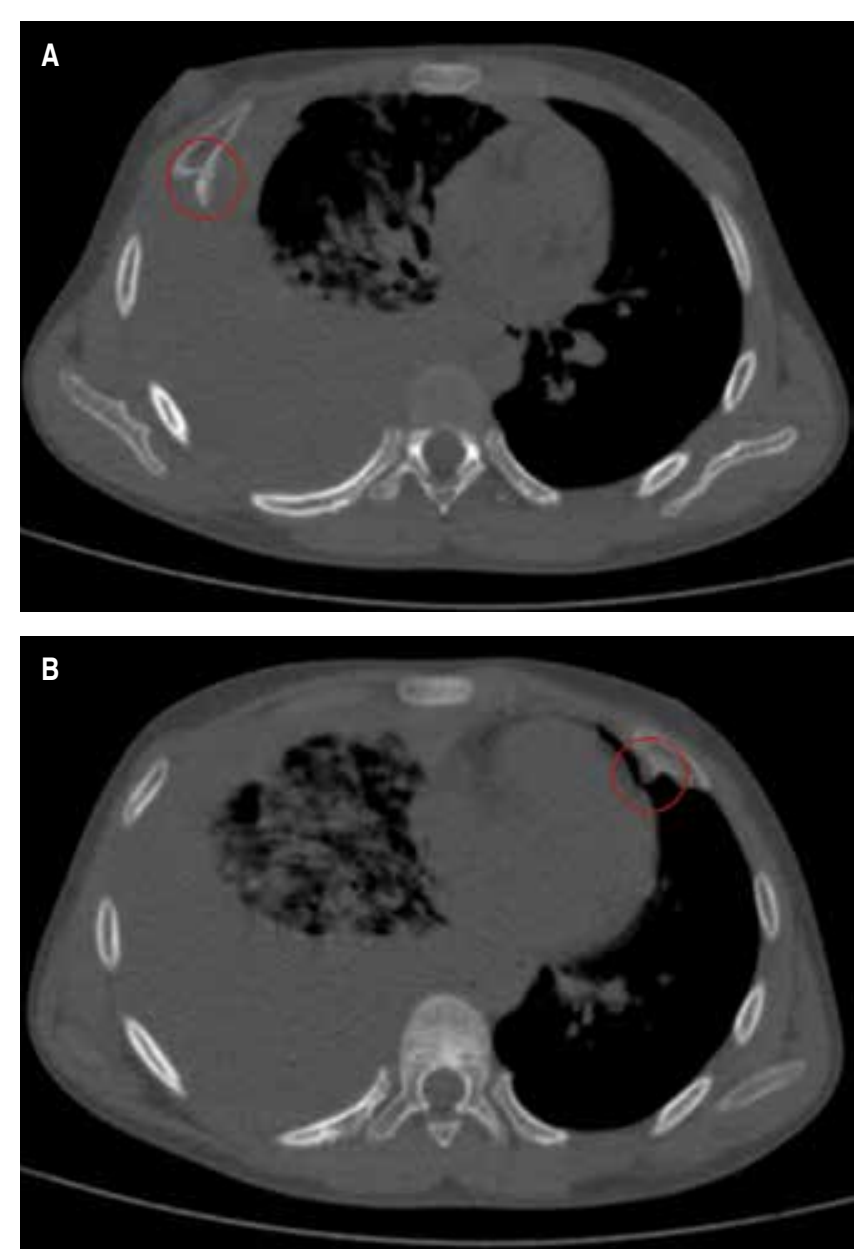

Figura 2: A) TAC simple de tórax en corte axial donde se observa una lesión exostósica en la quinta costilla de hemitórax derecho. B) Tomografía axial computarizada simple de tórax en corte axial donde se observa una lesión exostósica en la sexta costilla del hemitórax izquierdo.

Se envió la pieza quirúrgica al área de patología, donde se reportó el diagnóstico histopatológico de condromatosis (Figuras 4 y 5).

\section{DISCUSIÓN}

Un hemotórax espontáneo se define como un derrame pleural con un hematócrito mayor de 50\% del hematócrito en la sangre periférica en la ausencia de un trauma natural o iatrogénico que afecta el pulmón o el espacio pleural. ${ }^{1}$

Los datos sobre el hemotórax espontáneo se limitan a informes de casos y series. Es importante distinguir entre derrame pleural hemático y un hemotórax. El hematócrito de cualquier derrame hemático debe medirse para descartar hemotórax. El líquido pleural puede parecer similar a la sangre cuando el hematócrito es bajo, hasta $5 \%$. Un hemotórax se define cuando el hematócrito 
del derrame plural es mayor de $50 \%$ de la sangre del paciente. $^{2}$

Los osteocondromas son los tumores óseos benignos más frecuentes durante la niñez. ${ }^{3}$ Éstos surgen del desplazamiento supercostal del cartílago de la placa de crecimiento epifisario. El crecimiento persistente de este fragmento y su osificación endocondral subsecuente resulta en una excrecencia ósea subperióstica. Los ostecondromas tienen predilección por la región metafisaria de los huesos largos de las extremidades, pero han sido reportados en una variedad de otras localizaciones como la escápula, la pelvis, costillas, clavículas y vértebras. ${ }^{4}$

La exostosis hereditaria múltiple (EHM) es un trastorno autosómico dominante con una penetrancia variable, causado por la mutación de dos genes supresores de tumores EXT1 o EXT2, los pacientes presentan crecimiento de múltiples osteocondromas durante la niñez y la adolescencia, y con un cese típico durante la adultez. ${ }^{5}$ La EMH tiene una base genética. El gen de la enfermedad (EXT1) ha sido mapeado en el cromosoma 8q23-P24 mediante análisis de ligamiento en familias informativas.
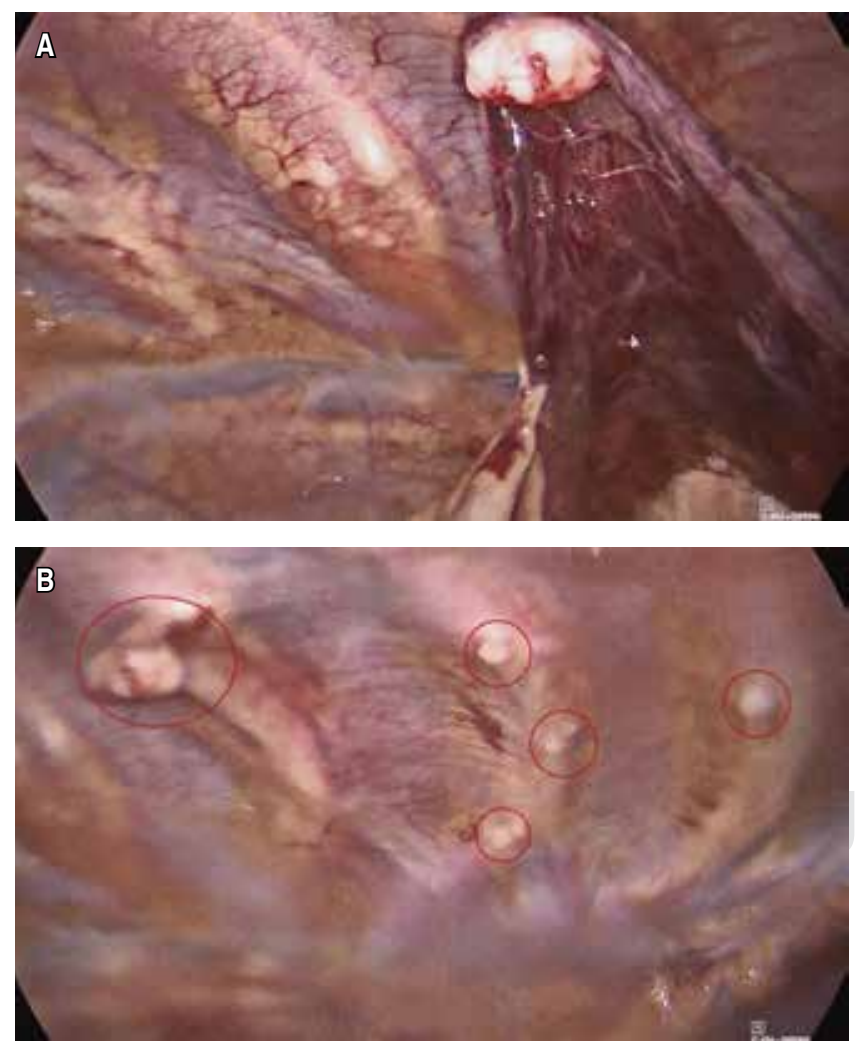

Figura 3: A) Vista toracoscópica de una lesión exostósica en la quinta costilla del hemitórax derecho, presumiblemente la causa del hemotórax, con un coágulo de aproximadamente $50 \mathrm{~cm}^{3}$ y sangrado en capa mínimo de la misma exostosis. B) Lesiones exostósicas múltiples en hemitórax derecho (resaltadas en círculos rojos).

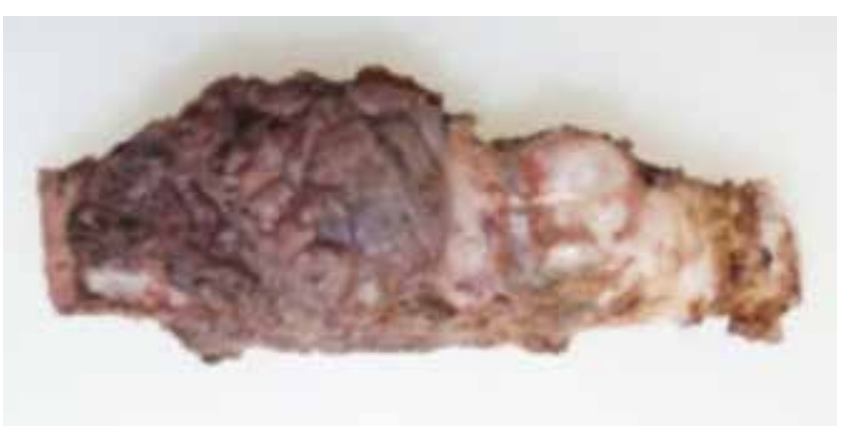

Figura 4: Cara externa de la pieza quirúrgica (cuarta costilla) con un condroma periosteal cercano al borde quirúrgico condral.

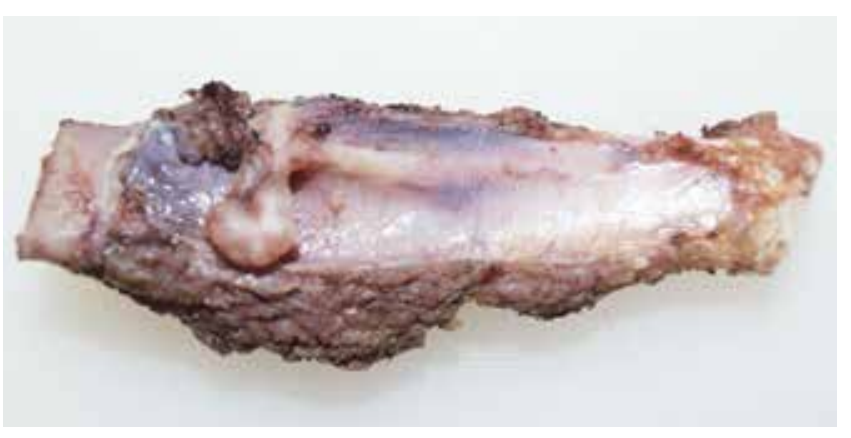

Figura 5: Cara interna de la pieza quirúrgica (cuarta costilla) con un condroma periosteal cercano al borde quirúrgico óseo.

En el mapeo genético aparece un segundo nicho o locus (EXT2) en el brazo corto del cromosoma 19, mediante la unión a un marcador de ADN de microsatélites en el locus D19S221.

Mientras algunas lesiones permanecen asintomáticas, estos tumores pueden causar deformidades angulares, afección de las articulaciones y daño a los tejidos blandos. $75 \%$ de los pacientes tiene deformidades obvias, más comúnmente en los antebrazos. ${ }^{5}$ Las exostosis costales representan una causa rara de hemotórax espontáneo o hemoneumotórax. ${ }^{6}$

Estos crecimientos óseos de las costillas pueden ser solitarios o múltiples en el síndrome de exostosis múltiple hereditaria. El mecanismo exacto del sangrado no es claro, pero se cree que se debe al daño de la pleura visceral y del parénquima pulmonar por el contacto directo con la exostosis relativamente afilada. De manera alternativa, el sangrado puede ser resultado de la ruptura de vasos dilatados asociados con irritación repetida de la pleura visceral. ${ }^{1}$

La colocación de un drenaje pleural es el tratamiento de primera línea para el hemotórax y su adecuada colocación es esencial para el drenaje efectivo del espacio pleural. Históricamente se han utilizado catéteres de grueso calibre cuando hay sospecha de hemotórax para 
prevenir la obstrucción por coagulación de la sangre. Las indicaciones tradicionales para intervención quirúrgica en un hemotórax agudo incluyen el drenaje inicial de 1,500 $\mathrm{mL}$ por la sonda pleural o drenaje de más de $200 \mathrm{~mL}$ por cuatro horas consecutivas. Sin embargo, los parámetros fisiológicos y las condiciones del paciente deben dirigir la intervención quirúrgica más que un volumen absoluto o el gasto por la sonda.?

La mejor opción de tratamiento para las exostosis es la escisión quirúrgica o curetaje. La toracoscopia permite una buena visualización para la evacuación del hematoma y el tratamiento de las exostosis. ${ }^{4}$

\section{CONCLUSIONES}

Es importante recalcar la importancia de tener una alta sospecha de esta entidad en pacientes con inicio súbito de dificultad respiratoria y antecedentes heredofamiliares de osteocondromas, ya que esto mejorará el pronóstico de nuestro paciente. Si bien los osteocondromas son entidades benignas, las complicaciones pulmonares asociadas a esta patología sí pueden llegar a tener repercusiones graves. El tratamiento siempre deberá enfocarse en la evacuación del hemotórax y la resolución quirúrgica de la exostosis.

\section{REFERENCIAS}

1. Morgan CK, Bashoura L, Balachandran D, Faiz SA. Spontaneous hemothorax. Ann Am Thorac Soc. 2015;12(10):1578-1582. https:// doi.org/10.1513/annalsats.201505-305cc.

2. Broderick SR. Hemothorax: Etiology, diagnosis, and management. Thorac Surg Clin. 2013;23(1):89-96, vi-vii. https://doi.org/10.1016/j. thorsurg.2012.10.003.

3. Mann E, Kaafarani HMA, Cassidy C, Chwals WJ, Jackson CC. Spontaneous hemothorax in multiple exostoses: a case report and review of literature. J Laparoendosc Adv Surg Tech A. 2011;21(6):575-577. https://doi.org/10.1089/lap.2010.0494.

4. Sainte $S$, Decaluwé $H$, Vanbrabant P. Spontaneous hemothorax, a rare face of vertebral osteochondroma. J Emerg Med. 2017;52(6):e225e228. https://doi.org/10.1016/j.jemermed.2017.01.026.

5. Cammarata-Scalisi F, Sánchez-Flores R, Stock-Leyton F, LabradorChacón N, Cammarata-Scalisi G. Exostosis múltiple hereditaria. Reporte de un caso y diagnóstico diferencial de las encondromatosis. Act Ortop Mex. 2012;26(6):388-392.

6. Bini A, Grazia M, Stella F, Petrella F. Acute massive haemopneumothorax due to solitary costal exostosis. Interact Cardiovasc Thorac Surg. 2003;2(4):614-615. https://doi.org/10.1016/s1569-9293(03)00122-1.

7. Pham-Duc ML, Reix P, Mure PY, Pracros JP, Moreux N, Bellon G. Hemothorax: an unusual complication of costal exostosis. J Pediatr Surg. 2005;40(11):e55-e57. https://doi.org/10.1016/j.jpedsurg.2005.07.060.

Conflicto de intereses: Los autores declaran no tener conflicto de intereses. 